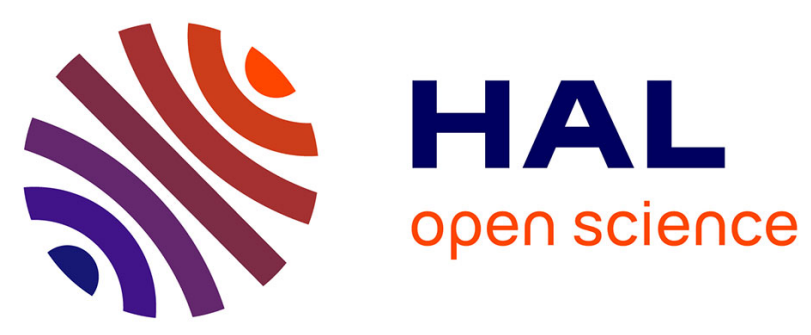

\title{
Resource Allocation for Real Time Services in LTE Networks: Resource Allocation Using Cooperative Game Theory and Virtual Token Mechanism
}

\author{
Mauricio Iturralde, Tara Ali-Yahiya, Anne Wei, André-Luc Beylot
}

\section{To cite this version:}

Mauricio Iturralde, Tara Ali-Yahiya, Anne Wei, André-Luc Beylot. Resource Allocation for Real Time Services in LTE Networks: Resource Allocation Using Cooperative Game Theory and Virtual Token Mechanism. Wireless Personal Communications, 2013, vol. 72 (n 2), pp. 1415-1435. 10.1007/s11277013-1086-z . hal-01130003

\section{HAL Id: hal-01130003 https://hal.science/hal-01130003}

Submitted on 11 Mar 2015

HAL is a multi-disciplinary open access archive for the deposit and dissemination of scientific research documents, whether they are published or not. The documents may come from teaching and research institutions in France or abroad, or from public or private research centers.
L'archive ouverte pluridisciplinaire $\mathbf{H A L}$, est destinée au dépôt et à la diffusion de documents scientifiques de niveau recherche, publiés ou non, émanant des établissements d'enseignement et de recherche français ou étrangers, des laboratoires publics ou privés. 


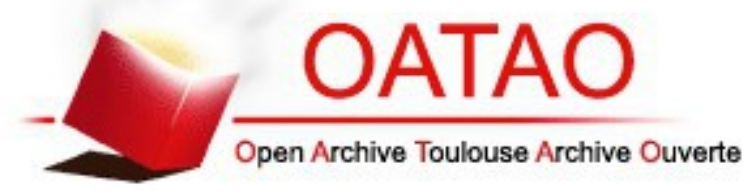

\section{Open Archive TOULOUSE Archive Ouverte (OATAO)}

OATAO is an open access repository that collects the work of Toulouse researchers and makes it freely available over the web where possible.

This is an author-deposited version published in : http://oatao.univ-toulouse.fr/ Eprints ID : 12394

To link to this article : DOI :10.1007/s11277-013-1086-Z

URL : http://dx.doi.org/10.1007/s11277-013-1086-Z

To cite this version : Iturralde, Mauricio and Ali-Yahiya, Tara and Wei, Anne and Beylot, André-Luc Resource Allocation for Real Time Services in LTE Networks: Resource Allocation Using Cooperative Game Theory and Virtual Token Mechanism. (2013) Wireless Personal Communications, vol. $72\left(\mathrm{n}^{\circ} 2\right)$. pp. 1415-1435. ISSN 0929-6212

Any correspondance concerning this service should be sent to the repository administrator: staff-oatao@,listes-diff.inp-toulouse.fr 


\title{
Resource Allocation for Real Time Services in LTE Networks: Resource Allocation Using Cooperative Game Theory and Virtual Token Mechanism
}

\author{
Mauricio Iturralde · Anne Wei • Tara Ali-Yahiya • \\ André-Luc Beylot
}

\begin{abstract}
The LTE specifications provide QoS for multimedia services with fast connectivity, high mobility and security. However, 3GPP specifications have not defined scheduling algorithms to exploit the LTE characteristics to support real time services. In this article we propose a two level scheduling scheme composed by cooperative game theory, a virtual token mechanism, and the well known algorithms EXP-RULE and Modified-Largest Weighted Delay Firs (M-LWDF) in downlink system. By using cooperative game theory such as bankruptcy game and Shapley value, the proposed mechanism works by forming coalitions between flow classes to distribute the bandwidth fairly among all of them. Both algorithms EXP-RULE and M-LWDF have been modified to use a virtual token mechanism to improve their performance, giving priority to real time flows. By taking the arrival rate of packets into account, the proposed mechanism partially included in previous schedulers has been adapted to this work to increase remarkably the performance of the resource allocation for real time flows. The performance evaluation is conducted in terms of system throughput, Packet loss ratio, total cell spectral efficiency, delay and fairness index.
\end{abstract}

Keywords Wireless networks · Quality of service - Long term evolution · Cooperative game theory $\cdot$ Shapley value $\cdot$ Scheduling algorithms

M. Iturralde $(\varangle) \cdot$ A.-L. Beylot

Université de Toulouse, IRIT/ENSEEIHT, Toulouse, France

e-mail: mauricio.iturralde@enseeith.fr

A.-L. Beylot

e-mail: andre-luc.beylot@enseeiht.fr

A. Wei

CNAM, Laboratoire cédric, Paris, France

e-mail: anne.wei@cnam.fr

T. Ali-Yahiya

Université Paris Sud 11, LRI, Paris, France

e-mail: tara.ali-yahiya@u-psud.fr 


\section{Introduction}

In conjunction with the useful growth of Internet, multimedia and RT services, Long Term Evolution (LTE) technology has been proposed to perform this ambitious task. LTE uses Orthogonal Frequency Division Multiple Access (ODFMA) in the downlink. OFDMA divides the frequency band into a group of mutually orthogonal subcarriers, thereby improving the system capabilities by providing high data rates, supporting multi-user diversity and creating resistance to frequency selective fading of radio channels. The Quality of Service (QoS) of LTE must be satisfied by giving users the optimal balance of utilization and fairness. Packet scheduling is one of the Radio Resource Management (RRM) mechanisms which is responsible for intelligent selection of flows for using the available resources such that specified performance metrics are satisfied. The need to support diverse QoS requirements of different applications whilst maximizing throughput is one of the major challenges in the design of packet scheduling algorithms in the downlink 3GPP LTE system. Real Time services need a high QoS level. This task can be performed by maintaining a desirable throughput, by ensuring a fair allocation and minimizing the packet loss and delay. Non-Real Time (NRT) services must have a minimum bit-rate. To satisfy this demand, several packet scheduling algorithms have been proposed Maximum-Largest Weighted Delay First (M-LWDF), Proportional Fair (PF) and Exponential Rule (EXP-RULE) [1,5,21]. In the above mentioned schedulers, each connection is assigned a priority value based on certain criterion, and the connection with the highest priority is scheduled at each Transmission Time Interval (TTI). Given that multimedia services such as video and VoIP are becoming the most important applications in telecommunications technology, this work adapts a scenario to use our proposed downlink scheduling scheme to improve their performance.

Overview of previous works. In the evolution of mobile cellular technologies generations, several mobile network technologies have born such as High-Speed Downlink Packet Access (HSDPA), Universal Mobile Telecommunications System (UMTS) and High Data Rate (HDR) systems. To guarantee an optimal QoS, several packet schedulers have been proposed such as PF, M-LWDF and EXP-RULE [1,4,12,24].

In [1] M-LWDF is presented as a potential scheduler for streaming services over HSDPA because it incorporates in the priority computation the time delay of packets, and the user instantaneous channel quality. In [12] PF algorithm has been introduced to perform the allocation over Code Division Multiple Access-High Data Rate (CDMA-HDR) systems. It has been shown by simulation that by taking advantage of the inherent multi-users diversity of a mobile wireless system which is the heart of the PF mechanism, the system throughput can be improved. However this algorithm does not account for delays of packet and can result in poor delay performance. Knowing that PF is not appropriate for a mixture of RT and NRT services, EXP-RULE has been introduced and potentially characterized as throughput optimal in [23].

HDR, HSDPA have a characteristic in common with LTE, all of them need to satisfy the QoS for high data bit rate applications e.g. video. Due to this common aspect, at the moment the resource allocation topic in downlink system over LTE has been treated based on existent schedulers used in the early mentioned technologies.

As early mentioned, the performance of several packet schedulers such as M-LWDF and EXP-RULE has been tested and proposed over OFDMA [3,13,21]. Both algorithms have been introduced to perform resource allocation in high data rate systems specifically focused on multimedia services. In [3] both algorithms have been compared and results show that M-LWDF performs a better system throughput as compared to EXP/PF for lower loads. 
On the other hand EXP/PF maintains a sustained performance for higher loads prioritizing RT services.

Additionally, other interesting scheduling methods for resource allocation in LTE have been proposed. In [22] authors propose an algorithm based on delays prioritizing for video streaming applications by utilizing user instantaneous downlink SNR values as well as its packet delay information. Results show an improvement of metrics compared to M-LWDF. In [19] a two-level scheduling algorithm for downlink is introduced. The proposed scheme is focused on RT services resource allocation helped by discrete time linear control theory. Authors consider the NRT flows allocation by using PF algorithm for best effort services.

Cooperative game theory has been introduced in economics as a formal model where groups of players form coalitions, to distribute the joint profits among their coalition. In [31] a resource allocation adapted to cooperative game theory for LTE downlink is presented. Authors use Nash Bargaining Solutions (NBS) to attack the fairness issue in resource allocation by finding an optimal fair subcarrier allocation. However, there are some well known constraints when using this method, the most common issue is the high complexity of NBS. When performing a NBS there exist two possible scenarios such as 2-person and n-person games. To avoid the complexity that n-person game carries on when the number of players is greater than two, most of authors have chosen the 2-person game $[8,17,30]$. This also implies that the authors have been forced to use other algorithms to divide the number of players in subsets of 2 elements. Also it is important to highlight that the vast majority of proposed schemes based on game theory are not focused on RT services performing.

Transferable Utility (TU) game is a scenario where one player can losslessly transfer part of its utility to another player. As part of TU game, bankruptcy problem is a distribution problem involving the allocation of a given amount of a perfectly divisible good among a group of agents. For doing so, Shapley value concept is presented as an alternative to NBS aiming to reach the same fairness level than NBS but presenting a low complexity level.

In previous works, Shapley value has been used to efficiently carry out bandwidth division. In [15] Shapley value has been proposed to perform resource allocation in $4 \mathrm{G}$ heterogeneous wireless networks. By adapting the "fairness" virtue of game theory concept, an improvement of resource allocation performance has been found. In [14] Shapley value has been used to compose bandwidth constraints in two bandwidth constraint models. It has been proven that game-based allocations can achieve greater efficiency in bandwidth sharing. In [10] we have adapted the Shapley value to perform a resource allocation focused on RT services in LTE networks. By our proposed scheme we have shown an improvement of performance and trade-off between throughput gain and fairness index for RT services.

Resource allocation by token based mechanisms is an interesting method to improve resource allocation schedulers. This technique using token queues, aims to grant equanimity in resource allocation between RT and NRT services. In [4] and [24] the EXP rule has been modified to be used in conjunction with virtual token mechanism to perform resource allocation for mixed service in High Data Rate (HDR) systems. Both works attempt to guarantee minimum throughput for NRT services. It has been shown in [4] that by adapting the EXP rule by using a token queue mechanism over HDR, a mixture of RT and NRT services can be supported with high efficiency. In our previous work [9] we evaluated the performance of EXP/PF and M-LWDF algorithms adapted to use a virtual token mechanism in a scenario for RT services over LTE networks. Consequently, we found that EXP/PF can achieve optimal results specially for VoIP and Video flows.

Contribution of the paper. The main contribution of our paper is a mechanism proposed specifically to improve the resource allocation for RT services in LTE networks in downlink 
system. As mentioned in the precedent section, several methods have been proposed but only few of them barely focus on RT services. In our work we use some well know techniques for resource allocation such as EXP-RULE algorithm, MLWDF algorithm and game theory as well, but wisely combined to make up an algorithm which presents a considerable improvement compared to other proposed solutions. Our algorithm has as characteristics robustness, a low complexity when performing game theory, a suitable trade off between throughput and fairness becoming like this a smart option to be set up in LTE.

For doing so, our mechanism adapts a game theory negotiation in bankruptcy games and the Shapley value to perform an efficient and fair bandwidth distribution among flow classes. Considering that, scheduling rules for multimedia services such as EXP-RULE and M-LWDF have been proposed to carry out the resource allocation in LTE, our proposed scheme performs a resource allocation focused on combining the early mentioned scheduling rules with a virtual token mechanism and cooperative game theory, to improve the resource allocation scheduling in downlink system. By combining the "robustness" of virtual token method and the "fairness" property which is the main attribute presented by the Shapley value, our proposed method aims to combine both characteristics to make up a robust scheduler with the capacity of performing a high throughput performance without neglecting fairness among flows.

Structure of the paper. This article is organized as follows. Section 2 explains the downlink system model in LTE, Sect. 3 describes the aforementioned cooperative game theory and its adaptation to resource allocation. Section 4 presents the aforementioned well known scheduling algorithms and the virtual token method. Section 5 exposes our resource allocation scheduling scheme. In Sect. 6, a simulation environment scenario is presented, where the traffic model is described and a numerical result analysis is exposed. Sect. 7 concludes this paper.

\section{Downlink System Model}

The QoS aspects of the LTE downlink are influenced by a large number of factors such as: Channel conditions, resource allocation policies, available resources, delay and sensitive/insensitive traffic. In LTE the resource that is allocated to a user in the downlink system, contains frequency and time domains, and is called resource block. The architecture of the 3GPP LTE system consists of several base stations called "eNodeB" where the packet scheduling is performed along with other Radio Resource Management (RRM) mechanisms (Fig. 1).

The entire bandwidth is divided into $180 \mathrm{kHz}$, physical Resource Blocks (RB's), each one lasting $0.5 \mathrm{~ms}$ and consisting of 6 or 7 symbols in the time domain, and 12 consecutive subcarriers in the frequency domain. The resource allocation is realized at every TTI, that is exactly every two consecutive resource blocks. In this way, resource allocation is done on a resource block basis.

Users report their instantaneous downlink channel conditions (e.g signal-to-noise-ratio, SNR) to the serving eNodeB at each TTI. At the eNodeB, the packet scheduler performs a user selection priority procedure, based on criteria such as channel conditions, Head Of Line (HOL) packet delays, buffers status and service types. The eNodeB has a complete information about the channel quality by the use of Channel State Information (CSI). Packets arriving into the buffer at eNodeB, are time stamped and queued for transmissions based on a First-In First-Out (FIFO) scheme (by flow). For each packet in the queue at the eNodeB buffer, the head of line (HOL) is computed, and a packet delay is computed as well. 


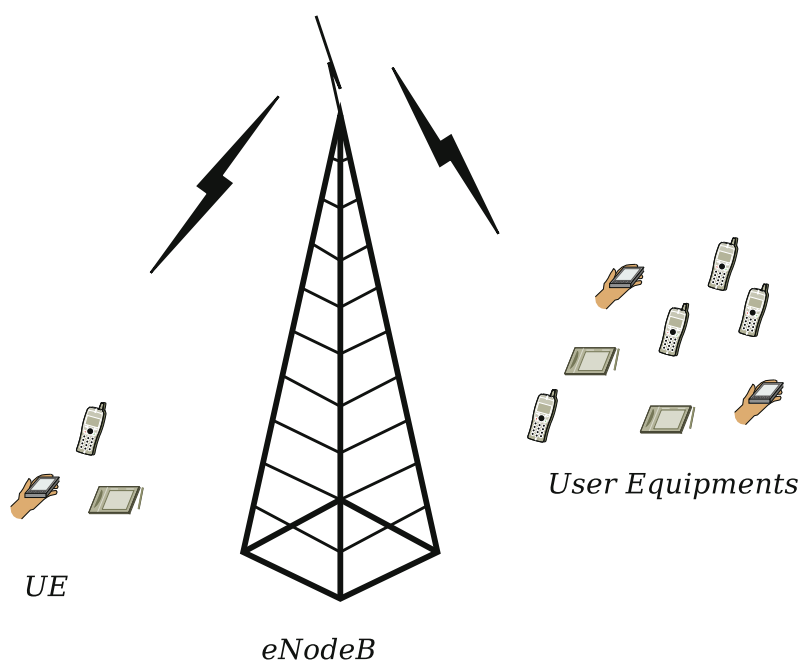

Fig. 1 LTE downlink system

\section{Cooperative Game Theory}

A cooperative game is a game where groups of players ("coalitions") may enforce cooperative behavior, hence the game is a competition between coalitions of players, rather than between individual players. This discipline concerns the behavior of decision makers (players) whose decisions affect each other. A cooperative game consist of a player list and characteristic function. Given a set of players $N$, the players should form a coalition to transfer benefits among them. Formally, a game is a pair $(N, v)$, where $N=\{1, \ldots, n\}$ is a finite set of players, $n=|N|$ and $v$ is a characteristic function $v: 2^{n} \rightarrow \mathbb{R}$ such as $v(0)=0$. Coalitions are subsets $S \subseteq N . N \backslash S$ denotes the complement set to $N$. In a game with $n$ players, there are $2^{n}$ possible coalitions.

\subsection{Transferable Utility (TU)}

Transferable utility is a term used in cooperative game theory and in economics. In a situation in which a finite set of players can obtain certain payoffs by cooperation can be described by a cooperative game with transferable utility or simply a TU game. Utility is transferable if one player or coalition of players can losslessly transfer part of its utility to another player or coalition of players. Such transfers are possible if the players have a common currency that is valued equally by all. In this case the assumption implies that irrespective of the division of the coalitional payoff, members of the coalition enjoy the same total utility.

\subsection{Bankruptcy Games}

Since the total utility (bandwidth in this approach) to be allocated is limited, this work is restricted to TU game. The analysis of bankruptcy situations tries to prescribe how to ration an amount of perfectly divisible resources among a group of players according to a profile of demands which, in the aggregate, exceeds the quantity to be distributed [16]. 
To illustrate a bankruptcy game, let us assume that a company becomes bankrupt. This company owns money to $n$ creditors and therefore, this money is needed to be divided among these creditors. Typically, the sum of the claims from the creditors is larger than the money of the bankrupt company. This conflicting situation introduces an $\mathrm{N}$-person game where the players of the game are seeking for the equilibrium point to divide money.

We model a bankruptcy situation by a triple $(N, C, g)$, where $N=\{1, \ldots, n\}$ is the set of players, $C \in \mathbb{R}_{+}$represents the benefit and $g=\left\{g_{1}, \ldots, g_{n}\right\} \in \mathbb{R}_{+}^{n}$ is the vector of claims of the players. In [16] for every bankruptcy problem $(N, C, g)$ an associated bankruptcy game $\left(N, v c_{g}\right)$ is defined.

Considering O'Neill approach, the value of a coalition $S$ is the part of the benefit that remains after paying the aggregated players in $N \backslash S$ all their bandwidth requirements, that is

$$
\begin{gathered}
v c_{g}(S)=\max \left\{C-\sum_{i \in N \backslash S} g_{i}, 0\right\} \\
v(N)=C
\end{gathered}
$$

\subsection{Shapley Value}

Shapley value is a Game Theory concept proposed by Lloyd Shapley [25] aiming to propose the fairest allocation of collectively gained profits between the several collaborative players. The basic criterion is to find the relative importance of every player regarding the cooperative activities.

To compute Shapley Value, let us define a function $\phi(v)$ as the worth or value of player $i$ in the game with characteristic function $v$. The Shapley value is the average payoff to a player if the player enters in the coalition randomly. The formula given by Shapley in [25] is:

$$
\phi_{i}(v)=\sum_{S \subseteq N} \frac{(|S|-1) !(n-|S|) !}{n !}(v(S)-v(S \backslash\{i\}))
$$

where $|S|$ indicates the number of players in the coalition, $n$ is the total number of players, $v(S)$ indicates the coalition utility including player $i$, and $v(S \backslash\{i\})$ indicates the coalition utility excluding player $i$.

The Shapley value is a very general method for equitable division. It is considered in economics as fairness standard, because it is a normative solution concept that prescribes a fair way to divide the gains from cooperation. The Shapley value is defined based on three axioms: symmetry, efficiency and additivity. The condition for efficiency is known as Pareto efficiency, and it gives guaranties that a player cannot obtain a better allocation without making another player allocation worse. Symmetry means that the player's final allocation does not depend on the order the players enter into the game. The symmetry property explains why the Shapley value is considered as a fairness standard. The additivity axiom specifies how the values of different games must be related to each other. If the allocation is defined for two independent games, so it is also valid for a composite game. In this work we focus on the TU game formalism.

\subsection{Resource Allocation Based Game Approach}

Consider a resource allocation problem where a finite divisible bandwidth capacity $C$ has to be divided among a finite set $N$ of flow classes. For each $i \in N$, each flow of a group of 
$k_{i}$ flows claims a bandwidth share $b_{i} \in \mathbb{R}_{+}$. Let $g_{i} \in \mathbb{R}_{+}$denotes the total class bandwidth claim. The vector of class resources claim are denoted as $g \equiv\left(g_{i}\right)_{i \in N}$, with $g_{i}=k_{i} b_{i}$. Each class represents a player. The benefit to be divided is the total resources at each TTI. When several classes share an amount of resource, we interpret them as forming a coalition, and the benefits should be distributed between the members of the coalition.

An arbitrator must divide the benefits among the players of the game efficiently. The arbitrator is the eNodeB in our case. It makes a fair resource blocks division among classes. A new re-distribution of resources is performed at each TTI. When a new class joins the groups and claims for resources, the arbitrator must initiate a new game to re-distribute bandwidth, this is performed in the next TTI. The inter-class division should be done using some fairness criteria, considering the different flows needs. Each class, having its corresponding bandwidth chunk, must divide the resource among their flows.

We consider a dynamic allocation process, and the number of flows in each class is variable. In a bandwidth allocation game, the classes represent the players who benefits from capacity $C$. All the classes forms a coalition to get the benefit $C$. Under-loaded classes cooperate with overload classes, giving way unused capacity.

\section{Scheduling Algorithms}

Resource allocation among different flows in wireless networks is performed by several scheduling policies such as channel gain, average rate, and packets arrival delay. For elastic flows, Proportional fairness (PF) is a good choice due to its capacity to allocate selecting users by their channel state. For RT services, algorithms based on packet delay such as M-LWDF have been proposed. For mixed flows, RT flows and NRT flows, algorithms as EXP/PF or EXP rule have also been proposed. In virtue of this work aims to perform only RT flows, the scheduling algorithms under consideration are specifically M-LWDF and EXP-RULE.

\subsection{Proportional Fair (PF)}

The Proportional Fair algorithm [5] is a very suitable scheduling option for NRT traffic. It assigns radio resources taking into account both the experienced channel quality and the past user throughput. The goal is to maximize the total network throughput and to guarantee fairness among flows. See (Eq. 3)

$$
j=\frac{\mu_{i}(t)}{\overline{\mu_{i}}}
$$

where $\mu_{i}(t)$ denotes the data rate corresponding to the channel state of the user $i$ at time slot $t, \overline{\mu_{i}}$ is the mean data rate supported by the channel.

\subsection{Maximum-Largest Weighted Delay First (M-LWDF)}

M-LWDF is an algorithm designed to support multiple RT data users in CDMA-HDR systems[1]. It supports multiple data users with different QoS requirements. This algorithm takes into account instantaneous channel variations and delays in the case of video service for instance.

The M-LWDF scheduling rule tries to balance the weighted delays of packets and to utilize the knowledge about the channel state efficiently. At time slot $t$, it chooses user $j$ for 
transmission as follows: (Eq. 4)

$$
j=\max _{i} a_{i} \frac{\mu_{i}(t)}{\overline{\mu_{i}}} W_{i}(t)
$$

where $\mu_{i}(t)$ denotes the data rate corresponding to the channel state of the user $i$ at time slot $t, \overline{\mu_{i}}$ is the mean data rate supported by the channel, $W_{i}(t)$ is the HOL packet delay and $a_{i}>0, i=1, \ldots, N$, are weights, which define the required level of QoS. According to [2], a rule for choosing $a_{i}$, which works in practice, is $a_{i}=-\log \left(\delta_{i}\right) T_{i}$. Here $T_{i}$ is the largest delay that user $i$ can tolerate and $\delta_{i}$ is the largest probability with which the delay requirement can be violated.

\subsection{Exponential Rule (EXP-RULE)}

Exponential Rule is an algorithm that was developed to support multimedia applications in an Adaptive Modulation and Coding and Time Division Multiplexing (ACM/TDM) system, this means that a user can belong to a RT service or NRT service [11]. This algorithm has been designed to increase the priority of RT flows with respect to NRT ones. Exponential rule has evolved due to some proposed changes, for this work the chosen version is presented in [25].

At time slot $t$, the EXP-RULE chooses user $j$ for transmission as follows: (Eq. 5)

$$
j=\max _{i} \begin{cases}\exp \left(\frac{a_{i} W_{i}(t)}{1+\sqrt{\bar{W}}}\right) \frac{\mu_{i}(t)}{\overline{\mu_{i}}} & (\mathrm{RT}) \\ \frac{\mu_{i}(t)}{\overline{\mu_{i}}} & \text { (NRT) }\end{cases}
$$

where $\mu_{i}(t)$ denotes the data rate corresponding to the channel state of the user $i$ at time slot $t$ while $\overline{\mu_{i}}$ is the mean data rate supported by the channel, this becomes the proportional fair rule [5]. $a_{i}=6 / d_{i}$ where $d_{i}$ is the is the maximal delay target of the $t h$ users flow. $W_{i}(t)$ is the HOL packet delay. According to [21], the parameter $a_{i}$ can be set to $6 / d_{i}$ or $10 / d_{i}$ $\left(a_{i} \in\left[5 / d_{i}, 6 / d_{i}, 10 / d_{i}\right]\right.$ gives good performance).

\subsection{Token Queues Mechanism}

It is essential that, queue state information such as queue length and packet delay which is a reflection of traffic robustness, to be utilized in scheduling packets. On the other hand, since the queue state information is tightly connected to the QoS, wisely controlling queues is one of the most effective ways for QoS provisioning. As it is described earlier, EXP-RULE and M-LWDF make scheduling decisions based on the actual packet delays. However, we propose to modify those algorithms by combining them with a virtual token mechanism. For doing so, a virtual token queue is associated to each flow, into which tokens arrive at constant rate $r_{i}$, the desired guaranteed minimum throughput of flow $i$. Let us define $V_{i}(t)$ to be the delay of the head of line token in the flow $i$ token queue. Note that we do not need to maintain the token delays. As the arrival rates of tokens are constant, $V_{i}(t)$ can be estimated as indicated in (Eq. 6).

$$
V_{i}(t)=\frac{Q_{i}(t)}{r_{i}}
$$

Where $Q_{i}(t)$ is the token queue length (a counter value at time $t$ ). The value for $r_{i}$ is 1 in our simulation scenario, therefore, the same desired minimum throughput is given to all flows. 


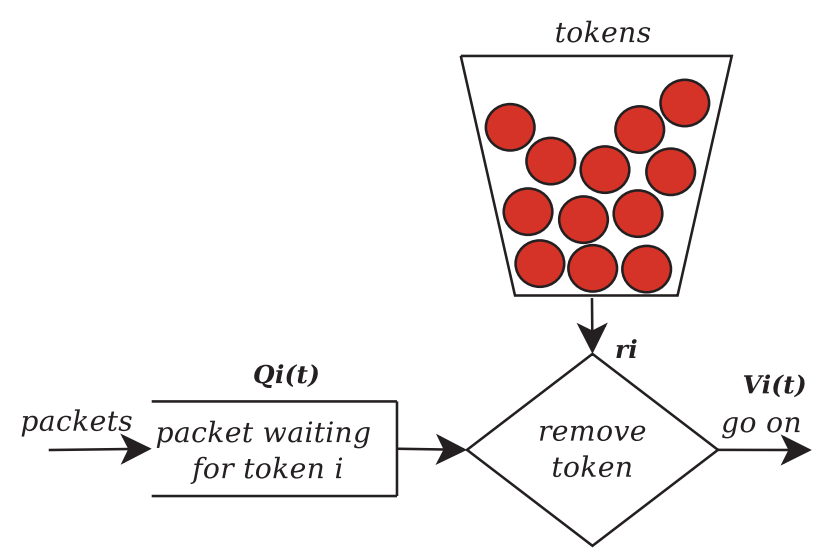

Fig. 2 Token queue mechanism

Then, we use M-LWDF and EXP-RULE algorithms with $W_{i}(t)$ being replaced by $V_{i}(t)$ respectively.

$$
\begin{aligned}
& j=\max _{i} a_{i} \frac{\mu_{i}(t)}{\overline{\mu_{i}}} V_{i}(t) \\
& j=\max _{i} \begin{cases}\exp \left(\frac{a_{i} V_{i}(t)}{1+\sqrt{\bar{W}}}\right) \frac{\mu_{i}(t)}{\overline{\mu_{i}}} & (\mathrm{RT}) \\
\frac{\mu_{i}(t)}{\overline{\mu_{i}}} & \text { (NRT) }\end{cases}
\end{aligned}
$$

After the service of a real queue, the number of tokens in the correspondent token queue is reduced by the actual amount of data transmitted (Fig. 2).

\section{Proposed Resource Allocation Mechanism}

The use of game theory has been proposed in several works to help the resource allocation process in wireless systems, but at this level it is important to highlight the issues that game theory carries on. As mentioned in the overview of previous works section the main problem when using game theory is its complexity level. Nash bargaining solutions presents two possible scenarios, 2-person game and n-person game. It is the n-person game scenario which presents the high complexity problem where the game complexity is directly proportional to the number of players which means that as more users enter in the game the complexity level increases [30]. The complexity level in n-person games has an exponential level. On the other hand a 2-person game presents a polynomial complexity solution which is lower than exponential, but the number of players is limited to two which is not real in wireless systems scenarios [17]. An n-person game will take too much time to process all the possible combinations among players which might spend far too much time to find the optimal solution, also it is important to take into account that n-person games may only have solutions all in irrational numbers.

In most cases in previous works the authors have adapted the 2-person game to be helped by another algorithm to form subsets of two players using like this the 2-person game scenario for all players when players are greater than two. Although this method reduce the complexity 


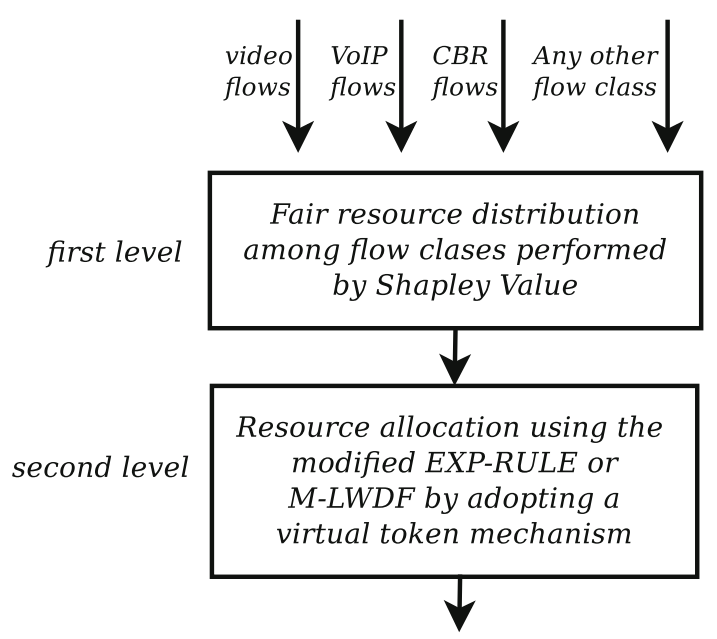

Fig. 3 Resource allocation levels

problem, there still exists complexity when using the algorithm to form the subsets of two players.

To avoid having these constraints we propose a resource allocation algorithm for a downlink system where the resource allocation is done at each TTI in two levels. On the first level a fair resource distribution among classes using Shapley value method is performed. At this level Shapley value performs a cooperation between players forming coalitions where it is so important to remark that in our game the players are the flow classes as explained in Subsect. 3.4. Having flow classes as players we can assume that the complexity of our game will not be high because the number of flow classes i.e. video, VoIP, Constant Bit Rate (CBR), Best effort etc will always be low.

After that, on the second level, having the proportion of resource destined to each class (video, VoIP, CBR, etc) a resource allocation is performed using EXP-RULE or M-LWDF, respecting the amount of resource that Shapley value has assigned to each class (Fig. 3). We have chosen EXP-Rule based on our previous performance study shown in [20], where we tested the virtual token mechanism with the well know algorithms such as PF, MLWDF and EXP-Rule where we found that the best performance is performed by EXP-Rule.

\subsection{First Level}

At this level a TU game is carried on, taking into account the parameters as shown in Table 1.

Table 1 Notation and description of variables for bankruptcy game and its adaptation to LTE scenario

\begin{tabular}{lll}
\hline Var & Bankruptcy game & Bandwidth allocation \\
\hline$n$ & Number of players & Number of flow classes \\
$C$ & Total benefit & Bandwidth capacity \\
$g_{i}$ & Player's benefit claim & Flow class bandwidth claim \\
\hline
\end{tabular}




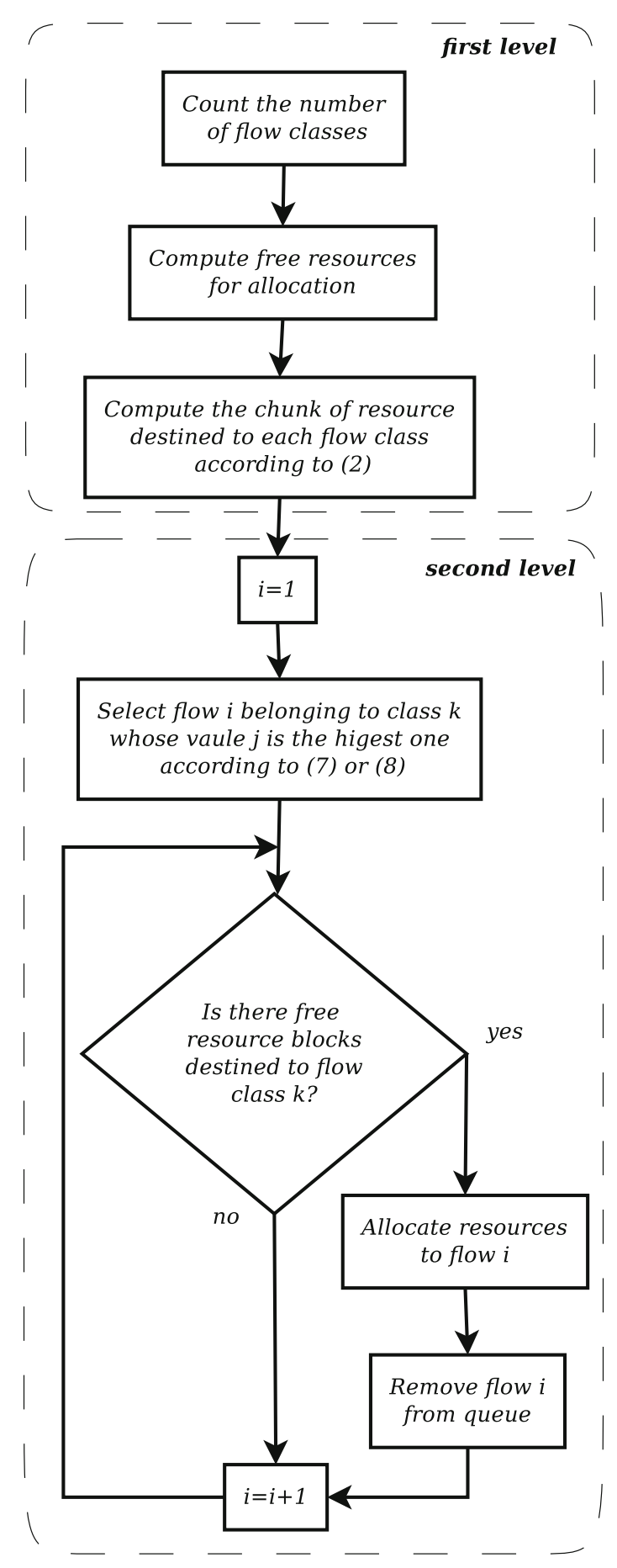

Fig. 4 Allocation algorithm performed at each TTI 
Bearing in mind Sect. 3 where resource allocation game approach was described, let us consider the following scenario to explain our resource allocation model.

Let us define three classes $A=$ video, $B=V o I P$ and $D=C B R$ as players in our scenario $N=\{A, B, D\}$. Consider $C=32 M b p s$ (50 Resource Blocks per TTI). The bandwidth required by a single flow of each class is $b=(242,8.4,2) \mathrm{kbps}$. The allocation is dynamic and depends on simultaneous flows quantity $K=\left(k_{A}, k_{B}, k_{D}\right)$. Thus, our bandwidth game is modeled as $\left(N ; v c_{g}\right)$ where $|N|=3$ and $v c_{g}(S)=\max \left\{C-\sum_{i \in N \backslash S} g_{i}, 0\right\}$, with $v(N)=C$. Developing the characteristic functions we have:

$$
\begin{array}{ll}
v c_{g}(1) & =\max \left\{32000-\left(8.4 k_{B}+2 k_{D}\right), 0\right\} \\
v c_{g}(2) & =\max \left\{32000-\left(242 k_{A}+2 k_{D}\right), 0\right\} \\
v c_{g}(3) & =\max \left\{32000-\left(242 k_{A}+8.4 k_{B}\right), 0\right\} \\
v c_{g}(1,2) & =\max \left\{32000-2 k_{D}, 0\right\} \\
v c_{g}(1,3) & =\max \left\{32000-8 k_{B}, 0\right\} \\
v c_{g}(2,3) & =\max \left\{32000-242 k_{A}, 0\right\} \\
v c_{g}(1,2,3) & =32000
\end{array}
$$

Thus, we go through to Shapley value (Eq. 2) to compute the resources related to each class depending on $K$.

\subsection{Second Level}

At this level, each flow class having its corresponding portion of resources, will distribute this portion to all flows belonging to it. The resource allocation is carried out using the aforementioned well known algorithms such as M-LWDF and EXP-RULE. These algorithms have been chosen based on their capacity of supporting RT services. By considering the HOL packet delays the early mentioned algorithms perform the best performance (but not the desired) for non-elastic flows. These algorithms have been modified to use the virtual token mechanism as presented in the last section. The virtual token mechanism forces the scheduler to stop to take into account the packet delays, to make the scheduling priority decision based on the largest packets queue which gives advantage to very large data flows. The scheduling mechanism is illustrated by Fig. 4.

\section{Simulation Environment}

To perform our resource allocation model, we define a scenario as follows. We use a single cell where there are $50 \%$ of users using video flows and $50 \%$ using VoIP flows. Users are constantly moving at speed of $3 \mathrm{kmph}$ in random directions (random walk). LTE-Sim simulator is used to perform this process [20]. LTE-Sim provides a support for radio resource allocation in a time-frequency domain. According to [20], in the time domain, radio resources are distributed every TTI, each one lasting $1 \mathrm{~ms}$. Furthermore, each TTI is composed by two time slot of $0.5 \mathrm{~ms}$, corresponding to 14 OFDM symbols in the default configuration with short cyclic prefix; 10 consecutive TTIs form the LTE Frame. Simulation parameters are shown in Table 2.

\subsection{Traffic Model}

A video service with $242 \mathrm{kbps}$ source video data rate is used in the simulation, this traffic is a trace-based application that sends packets based on realistic video trace files which are 
Table 2 LTE downlink simulation parameters

\begin{tabular}{lc}
\hline Parameters & Values \\
\hline Simulation duration & $100 \mathrm{~s}$ \\
Flows duration & $80 \mathrm{~s}$ \\
Frame structure & FDD \\
Radius & $1 \mathrm{~km}$ \\
Bandwidth & $10 \mathrm{MHz}$ \\
Slot duration & $0.5 \mathrm{~ms}$ \\
Scheduling time (TTI) & $1 \mathrm{~ms}$ \\
Number of RBs & 50 \\
Max delay & $0.1 \mathrm{~s}$ \\
video bit-rate & $242 \mathrm{kbps}$ \\
VoIp bit-rate & $8.4 \mathrm{kbps}$ \\
\hline
\end{tabular}

available on [29]. For VoIP flows, G.729 voice flows are generated by the VoIP application. In particular, the voice flow has been modeled with an ON/OFF process where the ON period is exponentially distributed with mean value $3 \mathrm{~s}$, and the OFF period has a truncated exponential probability density function with an upper limit of $6.9 \mathrm{~s}$ and an average value of $3 \mathrm{~s}$ [6]. During the ON period, the source sends 20 bytes sized packets every $20 \mathrm{~ms}$ (i.e., the source data rate is $8.4 \mathrm{kbps}$ ), while during the OFF period the rate is zero because the presence of a Voice Activity Detector is assumed. The fairness among users is measured using the Jain's fairness method [11].

The LTE propagation loss model is composed by 4 different models (shadowing, multipath, penetration loss and path loss)[28]

- Pathloss: $P L=128: 1+37: 6 \log (d)$ where $d$ is the distance between the UE and the eNodeB in $\mathrm{km}$.

- Multipath: Jakes model

- PenetrationLoss: $10 \mathrm{~dB}$

- Shadowing: log-normal distribution $($ mean $=0 \mathrm{~dB}$, standard deviation $=8 \mathrm{~dB})$

\subsection{Numerical Results}

This study seeks to combine Shapley value fairness property, a virtual token mechanism and the well known algorithms such as EXP-RULE and M-LWDF to improve the resource allocation scheduling in downlink. To better understand the obtained results, the following notation is used: "EXP-RULE" represents the non-modified exponential rule, "EXP-RULEVT-SH" represents the modified exponential rule using a virtual token mechanism combined with the Shapley value method, "M-LWDF" represents the classical M-LWDF scheduling algorithm, and "M-LWDF-VT-SH" represents the modified M-LWDF using a virtual token mechanism combined with the Shapley value method.

Throughput. Average throughput per video flow is shown in Fig. 5. When using EXP-RULEVT-SH the throughput increases up to $30 \%$ when the cell is loaded by 50 users, which means that the quality of video service is still acceptable for 50 users whereas that EXP-RULE supports only 33 users. By adopting the view that HOL is removed of EXP-RULE when it is modified by the virtual token mechanism, we can justify its throughput increase because 


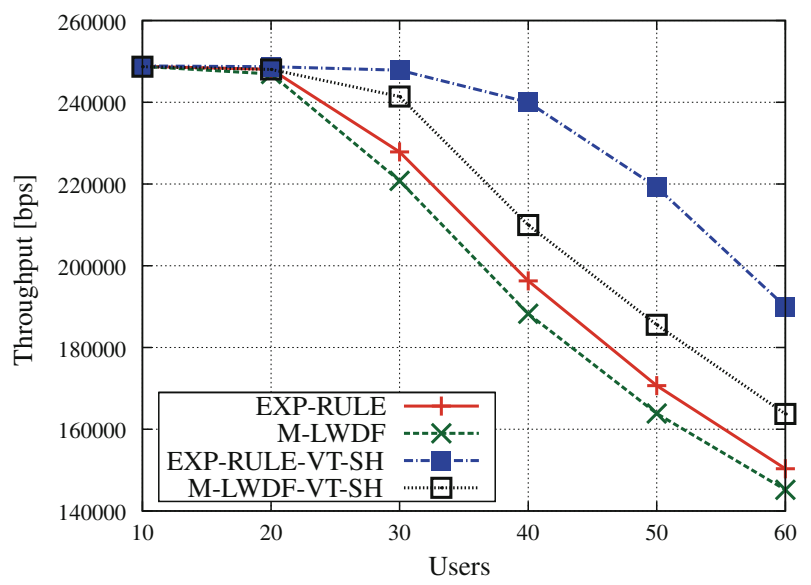

Fig. 5 Average throughput per video flow

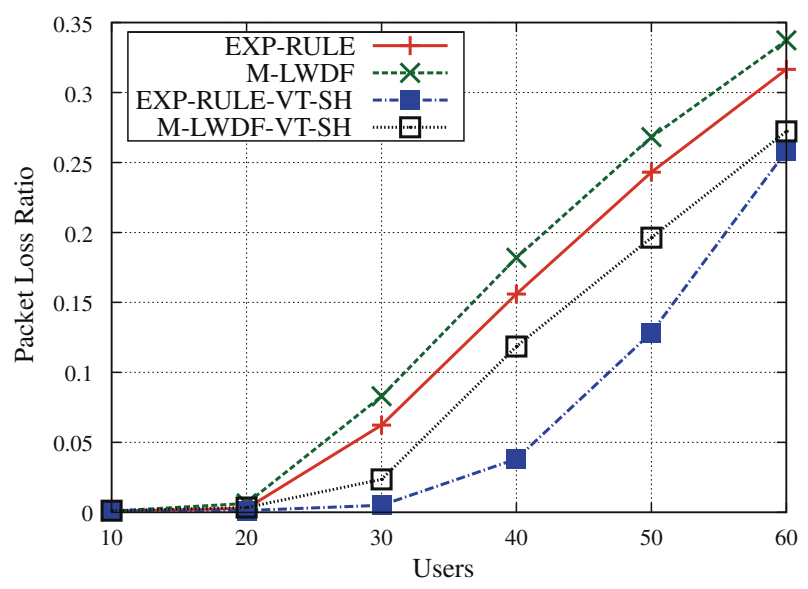

Fig. 6 Packet loss ratio for video flows

of the high bit-rate needed for video flows, therefore video flows have a higher quantity of tokens. On the other hand the Shapley value division guarantees resource allocation at each TTI for each flow class, like this we can assure a minimum bit-rate to flows that eventually have a very low quantity of tokens which is the weakness of virtual token method.

M-LWDF also shows a throughput increase performed by the proposed modifications. As expected M-LWDF-VT-SH does not reach a significant increase as EXP-RULE-VT-SH. M-LWDF-VT-SH guarantees an acceptable video service for 38 users, 6 users more than the non-modified M-LWDF-VT-SH, it means that M-LWDF-VT-SH presents a throughput increases up to $19 \%$.

There is not a significant variation of throughput for VoIP when using the modified and nonmodified schedulers utilized in this work. All presented schedulers show a good performance for VoIP flows, the throughput is maintained when the cell is totally loaded by 60 users. One possible explanation for this no-variation is the ON/OF periods implemented in the LTE-Sim VoIP model (Fig. 9).

Packet Loss Ratio. PLR for video flows decreases by up to $38 \%$ when using EXP-RULE-VTSH compared to EXP-RULE. M-LWDF-VT-SH also presents a PLR decrease by up to $19 \%$. 


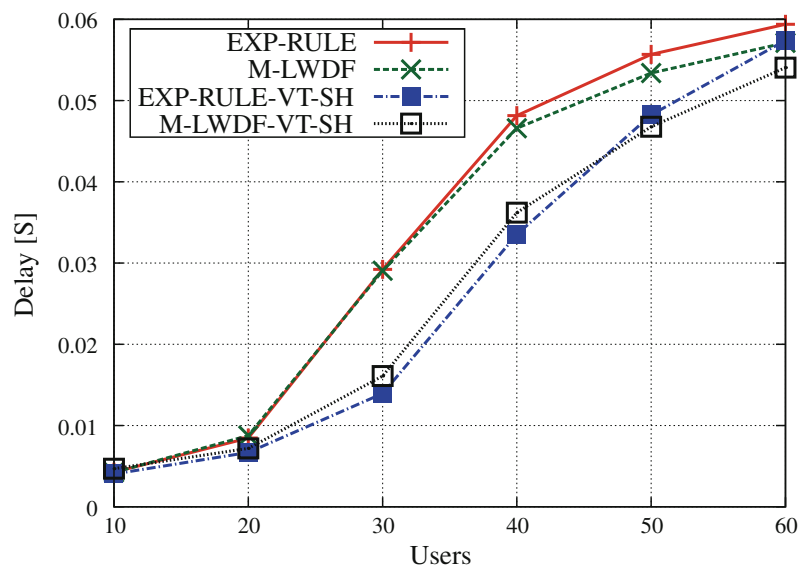

Fig. 7 Delay for video flows

Considering that to maintain an acceptable video flow service the PLR shall not overcome the $3 \%$, EXP-RULE-VT-SH presents the best performance maintaining 47 users under this range whilst M-LWDF-VT-SH maintains 38 users over this range (Fig. 6). For VoIP flows, EXPRULE-VT-SH presents an increase of PLR up to $74 \%$ compared to EXP-RULE. Although the PLR increase reached by EXP-RULE-VT-SH is considerable, it does not overcome the $1.2 \%$, this is still a good result considering that the accepted PLR for VoIP flows must be under the $3 \%$.

M-LWDF-SH-VT performs the best result of PLR, this value is maintained under $0.04 \%$ when the cell is totally loaded by 60 users. The decrease percentage presented by M-LWDFVT-SH is up to $160 \%$ compared to the classic M-LWDF (Fig. 10).

Delay. As seen in Fig. 7, the delay for video flows decreases by up to $4 \%$ when using EXPRULE-VT-SH compared to the non-modified EXP-RULE, on the other hand when using M-LWDF-VT-SH, the delay decreases by $6 \%$ when the cell is totally loaded by 60 users (Fig. 8, 9, 10, 11, 12).

For VoIP flows, there is a clear decrease of delay when the cell is totally loaded by 60 users. When using EXP-RULE-VT-SH the delay decreases by up to $16.9 \%$. M-LWDF-VT$\mathrm{SH}$ performs the best delay, it decreases considerably by $38 \%$ when the cell is loaded by 60 users compared to M-LWDF (Fig. 11).

Fairness Index. For video flows fairness index values has been computed (Fig. 7). As described above, the main Shapley value virtue is its fairness characteristic. Due to its implementation in the proposed scheme, the fairness value increases. When the cell is totally loaded by 60 users, EXP-RULE-VT-SH presents a fairness index increases up to $3.5 \%$ compared to EXP-RULE and M-LWDF-VT-SH shows a fairness index increase up to $2 \%$ compared to M-LWDF.

Total cell Spectral Efficiency. Figure 12 shows an improvement of spectral efficiency performed by the two modified algorithms. This rise is due to the video throughput increase above analyzed. When the cell is loaded by 60 users EXP-RULE-VT-SH presents a rise up to 


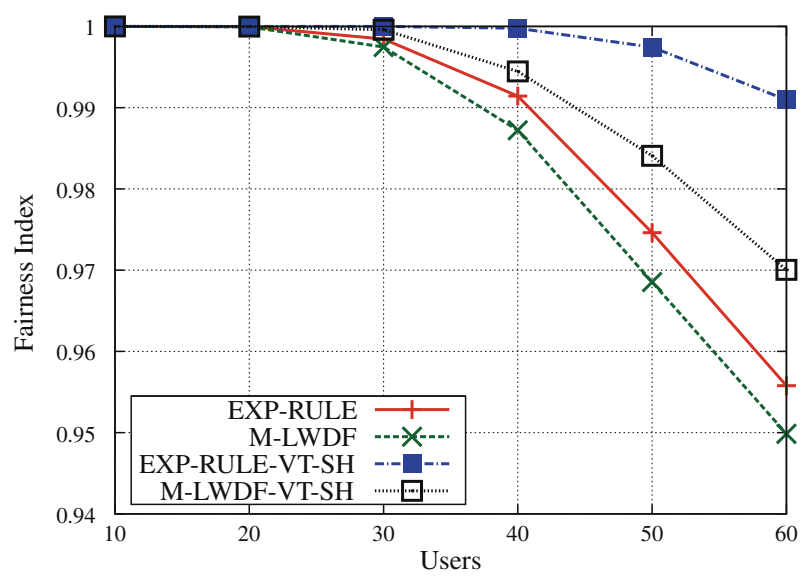

Fig. 8 Fairness index for video flows

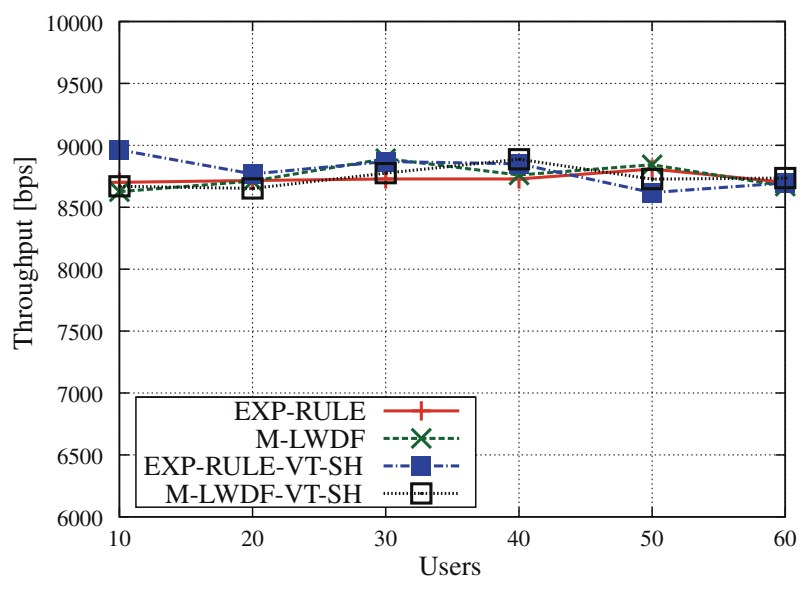

Fig. 9 Average throughput per VoIP flow

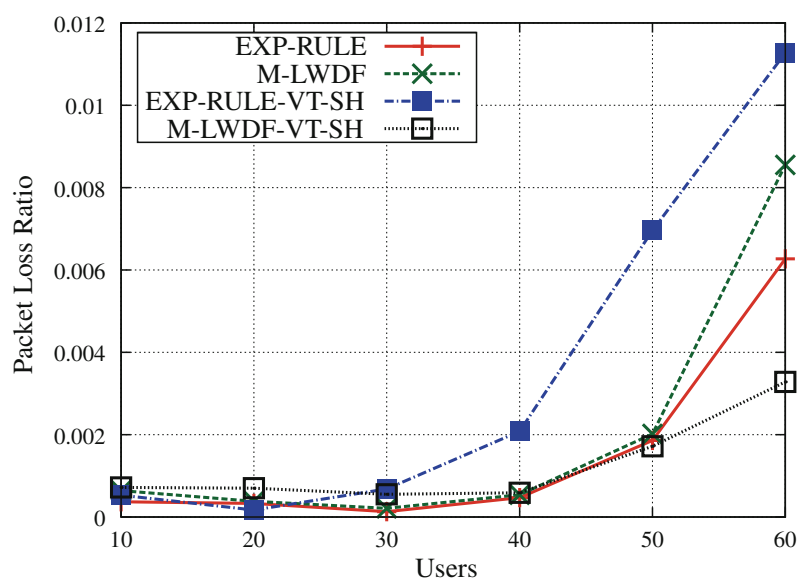

Fig. 10 Packet loss ratio for VoIP flows 


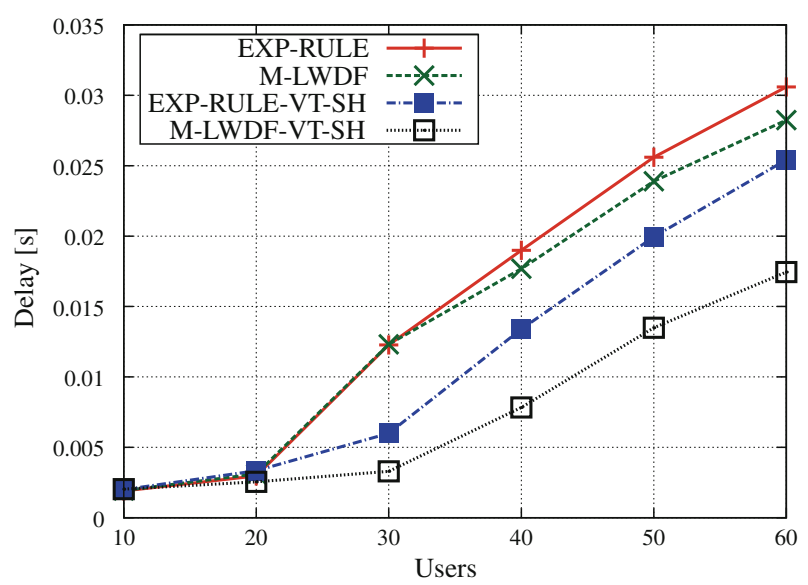

Fig. 11 Delay for VoIP flows

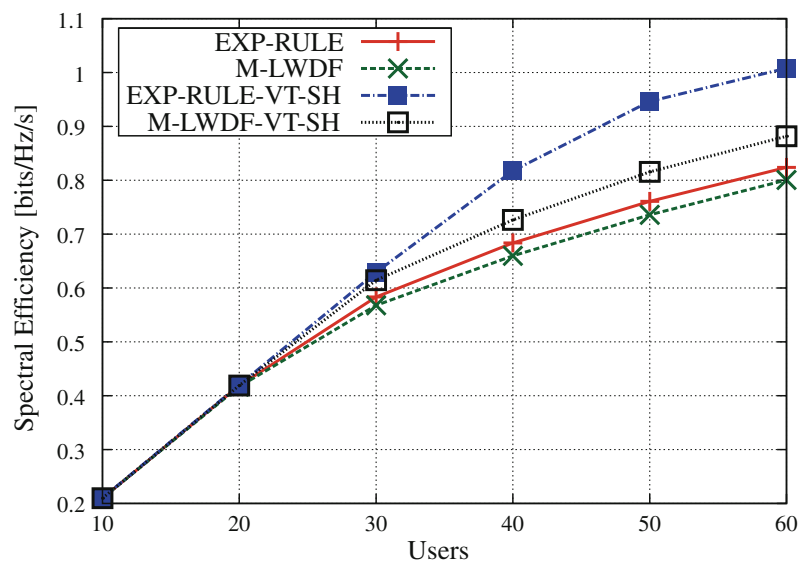

Fig. 12 Total spectral cell efficiency

Table 3 Comparison of results between modified scheduling algorithms

\begin{tabular}{llll}
\hline & Metric & EXP-RULE-VT-SH & M-LWDF-VT-SH \\
\hline Video & Throughput & Best & Better \\
& PLR & Best & Better \\
& Delay & Better & Better \\
& Fairness index & Best & Better \\
VoIP & Throughput & Good & Good \\
& PLR & Good & Best \\
& Delay & Better & Best \\
Spectral efficiency & & Best & Better \\
\hline
\end{tabular}


$22 \%$ compared to EXP-RULE. M-LWDF-VT-SH also presents a spectral efficiency increase up to $10 \%$ compared to M-LWDF. The best performance is reached by EXP-RULE-VT-SH.

To contrast the results obtained by simulations, a summary of results is presented in Table 3 where, both M-LWDF-SH-VT and EXP-RULE-VT-SH are compared to the non modified rules M-LWDF and EXP-RULE. Adjectives such as "good", "better" and "best" are used to carry out this comparison. "good" involves a competent or skilled performance, "better" connotes an improved performance and "best" represents the most excellent performance.

\section{Conclusions}

This study paper has focused attention on resource allocation in downlink system for RT services in LTE networks. We defined five performance metrics, namely throughput, PLR, fairness Index, delay and total cell spectral efficiency. With respect to these measures we can conclude that by modifying the presented scheduler algorithms such as EXP-RULE and M-LWDF to use a virtual token mechanism, it is possible to enhance their performance. Similar results have been obtained by combining the mentioned algorithms with the Shapley value method. A fusion of these two mechanisms improves the performance of both algorithms considerably by using the robustness of Virtual Tokens method, and fairness as the particular characteristic of Shapley value. Comparing the two mentioned modified algorithms to use the proposed scheme, we can assume that EXP-RULE-VT-SH presents the best improvement in performance. For constrains such as delay and PLR, M-LWDF-VT-SH presents a better performance than EXP-RULE-VT-SH however EXP-RULE-VT-SH values are into the expected range. Our proposed scheme is open to changes on the second level if there is found another scheduler that could perform better results than EXP-Rule. The proposed scheme allows a low complexity implementation, which is suitable for practical wireless systems. Future work could be focused in using game theory mechanisms to propose a fair distribution of resources in uplink system.

\section{References}

1. Ameigeiras, P., Wigard, J., \& Mogensen, P. (Sep. 2004). Performance of the m-lwdf scheduling algorithm for streaming services in hsdpa. In IEEE Transactions on vehicular technology conference vol. 2, pp. 999-1003 Los Angeles, USA.

2. Andrews, M., Kumaran, K., Ramanan, K., Stolyar, A., Vijayakumar, R., \& Whiting, P. (2001). Providing quality of service over a shared wireless link. IEEE Communications Magazine, 39(2), 150-154.

3. Basukala, R., Mohd Ramli, H., \& Sandrasegaran, K. (Nov. 2009). Perfomance analysis of EXP/PF and M-LWDF in downlink 3GPP TLE system. In IEEE first Asian himalayas conference, pp. 1-5 Kathmandu, Nepal.

4. Chang, K., \& Han, Y. (September 2002). QoS-based adaptive scheduling for a mixed service in HDR systems. In IEEE international symposium (PIMRC). vol. 4, pp. 1914-1918, Lisboa, Portugal.

5. Choi, J.-G., \& Bahk, S. (2007). Cell-throughput analysis of the proportional fair scheduler in the single-cell environment. IEEE Transactions on Vehicular Technology, 56(2), 766-778.

6. Chuah, C., \& Katz, R. H. (Apr. 2002). Characterizing packet audio streams from internet multimedia applications. In IEEE international communications conference (ICC) vol. 2, pp. 1199-1203 New York, USA.

7. Ekstrom, H. (2009). QoS control in the 3GPP envolved packet system. IEEE Communications Magazine, 47(2), 76-83.

8. Han, Z., Ji, Z., \& Liu, K. J. R. (2005). Fair multiuser channel allocation for OFDMA networks using Nash Bargaining solutions and coalitions. IEEE Transactions on Communications, 53(8), 1366-1376. 
9. Iturralde, M., Yahiya, T., Wei, A., \& Beylot, A. (Sep. 2011). Performance study of multimedia services using virtual token mechanism for resource allocation in LTE networks. IEEE vehicular technology conference (VTC), San Francisco, USA.

10. Iturralde, M., Yahiya, T., Wei, A., \& Beylot, A. (Sep. 2011). Resource allocation using shapley value in LTE networks. In IEEE international conference on personal, indoor and mobile radio communications (PIMRC), Toronto, Canada.

11. Jain, R., Chiu, D., \& Hawe, W. (1984). A quantitative measure of fairness and discrimination for resource allocation in shared computer systems. Digital EquipmentCorporation, Littleton, MA, DEC Rep., DECTR-301.

12. Jalali, A., Padovani, R., \& Pankaj, P. (May. 2000). Data throughput of CDMA-HDR a high efficiencyhigh data rate personal communication wireless system. In IEEE vehicular technology conference (VTC), Tokio, Japan.

13. Kim, K., Koo, I., \& Sung, S. (Oct 2004). Multiple qos support using m-lwdf in ofdma adaptive resource allocation. In IEEE local and metropolitan networks workshop (LANMAN). pp. 217-221. San Francisco, USA.

14. Meneses, B., Monteiro, J., \& Salgueiro, R. (October 2009). Defining bandwidth constraints with cooperative games. In IEEE international ultra modern telecommunications and workshops (ICUMT) conference vol. 1, pp. 1-8 St. Petersburg, Rusia.

15. Niyato, D., \& Hossain, E. (June 2006). A cooperative game framework for bandwidth allocation in $4 \mathrm{G}$ heterogeneous wireless networks. In IEEE international communications conference (ICC) vol. 9, pp. 4357-4363, Istanbul, Turkey.

16. O'Neill, B. (1982). A problem of rights arbitration from the Talmud. Mathematical Social Sciences, 2 , 345-371.

17. Park, H., \& van der Schaar, Mihaela. (2007). Bargaining strategies for networked multimedia resource management. IEEE Transactions on Communications, 55(7), 3496-3511.

18. Pedersen, K., Kolding, T., Frederiksen, F., Kovacs, I., Laselva, D., \& Mogensen, P. (2009). An overview of downlink radio resource management for UTRAN long-term evolution. IEEE Communications Magazine, 47(7), 86-93.

19. Piro, G., Grieco, L., Boggia, G., \& Camarda, P. (April 2010). A two-level scheduling algorithm for QoS support in the downlink of LTE cellular networks. In IEEE wireless conferece European (EW), Lucca, Italy.

20. Piro, G., Grieco, L., Boggia, G., Capozzi, F., \& Camarda, P. (2010). Simulating lte cellular systems: An open source framework. IEEE Transactions on Vehicular Technology, 60(2), 498-513.

21. Sadiq, B., Madan, R., \& Sampath, A. (2009). Downlink scheduling for multiclass traffic in LTE. EURASIP Journal on Wireless Communications and Networking, 2009, 1-18.

22. Sandrasegaran, K., Ramli, M., Adibah, H., \& Basukala, R. (Apr 2010). Delay-prioritized scheduling (DPS) for real time traffic in 3GPP LTE system. In IEEE Wireless communications and networking conference $(W C N C)$, Sydney, Australia.

23. Shakkottai, S., \& Stolyar, A. (2000). Scheduling for multiple flows sharing a time-varying chanel: The exponential rule. Bells Laboratories.

24. Shakkottai, S., \& Stolyar, A. (2004). Scheduling algorithms for a mixure of real time and non real time data in HDR. Bells Laboratories.

25. Shapley, L. S. (1953). A value for N-Person game. Annals of mathematics Studies, Princeton University press, 2, 307-317.

26. Tech. Specif. Group Radio Access Network 3GPP. Feasibility study for orthogonal frequency division multiplexing (OFDM) for UTRAN enhancement (Release 6)". Technical report, 3GPP TS 25.892.

27. Tech. Specif. Group Radio Access Network 3GPP. Medium access control (MAC) protocol specification (release 9)". Technical report, 3GPP TS 36.321.

28. Tech. Specif. Group Radio Access Network 3GPP. Physical layer aspect for evolved universal terrestrial radio access (utra) (release 7). Technical report, 3GPP TS 25.814.

29. Video trace library. http://trace.eas.asu.edu/

30. Yaacoub, E., \& Dawy, Z. (April 2009). A game theorical formulation for proportional fairness in LTE uplink scheduling. In IEEE Wireless Communications and Networking Conference (WCNC), pp. 1-5, 5-8 Budapest, Hungary.

31. Zhang, G., \& Zhang, H. (Nov 2008). Adapative resource allocation for downlink OFDMA networks using cooperative game theory. In IEEE Communication Systems Signal International Conference (ICCS) Guangzhou, China. 


\section{Author Biographies}

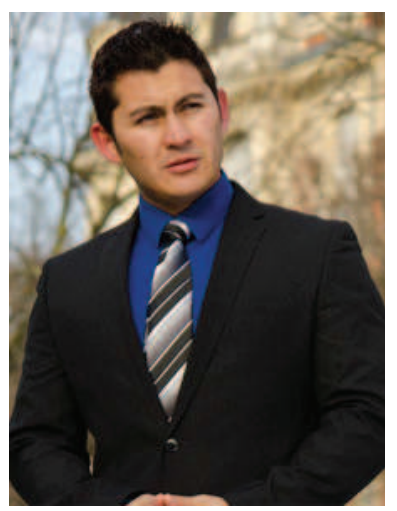

Mauricio Iturralde received his Engineer degree in Computer Science from Technical University of Ambato in 2007, Ambato, Ecuador. He received his master degree in Systems Information Security from University of Paris XII, (Val de Marne) in 2009, Paris, France. He is currently working toward his Ph.D. degree in computer science at University of Toulouse, IRIT/ENSEEIHT, Toulouse, France. His research interests include quality of service in wireless network and resource management.

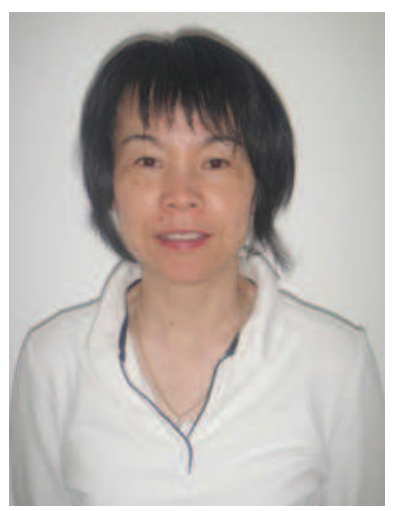

Anne Wei graduated from the Department of Electronic Engineering of the Shanghai University in 1986 and completed her Ph.D. in 1999 at Institute National des Telecommunications, France. After two years spent working for STERIA a computing system company and eight years as an Assistant professor at the University of Paris XII and two years as Professor at the University of Toulouse II, she is now a Professor at the CNAM Paris where she teaches computing science and networks. Her research interests include computer networks, post-3G mobile systems and especially security, QoS and mobility in wireless network communications.

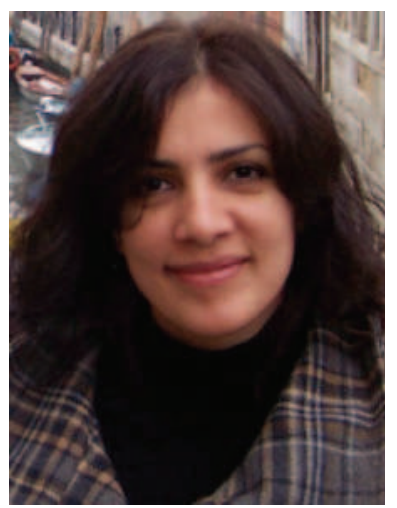

Tara Ali-Yahiya received her M.S. in Computer Science from Gaspard-Monge Institute in the University of Marne-la-vallée in 2004. She received her Ph.D. degree in network and computer science from the University of Paris VI, Paris, France, in 2008. She was post doctoral fellow in Telecom SudParis, Evry, France during 2009. Currently, she is associate professor at the University Paris Sud 11, Orsay, France. Her research interests include wireless networks, resource allocation under QoS, network planning and modeling, as well as performance evaluation. 


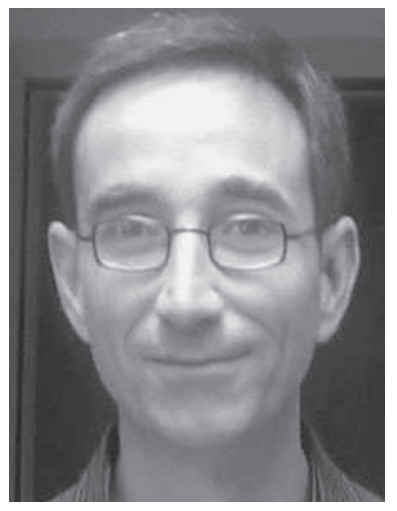

André-Luc Beylot received the Engineer degree from the Institut d'Informatique d'Entreprise, Evry, France, in 1989 and the Ph.D. degree in computer science from the University of Paris VI, Paris, France, in 1993. In January 2000, he received the Habilitation á? Diriger des Recherches from the University of Versailles, France. From September 1996 to August 2000, he was an Assistant Professor at PRiSM Laboratory, University of Versailles. Since September 2000, he has been a Professor at the Telecommunication and Network Department, National Polytechnics Institute of Toulouse (INPT/ENSEEIHT). Since September 2009, he leads the IRT Team of the IRIT Laboratory. His research interests are with the performance evaluation of communication networks, especially with regard to mobile, satellite, and vehicular networks. 